\title{
Nitric oxide (NO) elicits aminoglycoside tolerance in Escherichia coli but antibiotic resistance gene carriage and NO sensitivity have not co-evolved
}

\author{
Cláudia A. Ribeiro ${ }^{1}$. Luke A. Rahman ${ }^{1}$. Louis G. Holmes ${ }^{1} \cdot$ Ayrianna M. Woody ${ }^{1}$. Calum M. Webster ${ }^{1}$. \\ Taylor I. Monaghan ${ }^{1} \cdot$ Gary K. Robinson ${ }^{1} \cdot$ Fritz A. Mühlschlegel ${ }^{1,2,3} \cdot \operatorname{lan}$ B. Goodhead ${ }^{4} \cdot$ Mark Shepherd $^{1}$ (1)
}

Received: 14 May 2020 / Revised: 17 November 2020 / Accepted: 14 February 2021

(c) The Author(s) 2021

\begin{abstract}
The spread of multidrug-resistance in Gram-negative bacterial pathogens presents a major clinical challenge, and new approaches are required to combat these organisms. Nitric oxide (NO) is a well-known antimicrobial that is produced by the immune system in response to infection, and numerous studies have demonstrated that NO is a respiratory inhibitor with both bacteriostatic and bactericidal properties. However, given that loss of aerobic respiratory complexes is known to diminish antibiotic efficacy, it was hypothesised that the potent respiratory inhibitor NO would elicit similar effects. Indeed, the current work demonstrates that pre-exposure to NO-releasers elicits a $>$ tenfold increase in $\mathrm{IC}_{50}$ for gentamicin against pathogenic $E$. coli (i.e. a huge decrease in lethality). It was therefore hypothesised that hyper-sensitivity to NO may have arisen in bacterial pathogens and that this trait could promote the acquisition of antibiotic-resistance mechanisms through enabling cells to persist in the presence of toxic levels of antibiotic. To test this hypothesis, genomics and microbiological approaches were used to screen a collection of $E$. coli clinical isolates for antibiotic susceptibility and NO tolerance, although the data did not support a correlation between increased carriage of antibiotic resistance genes and NO tolerance. However, the current work has important implications for how antibiotic susceptibility might be measured in future (i.e. \pm NO) and underlines the evolutionary advantage for bacterial pathogens to maintain tolerance to toxic levels of NO.
\end{abstract}

Keywords Aminoglycoside $\cdot$ Escherichia coli $\cdot$ Nitric oxide $\cdot$ Respiration $\cdot$ Antibiotic resistance

\section{Introduction}

For the last eight decades, antibiotics have been used to combat infectious diseases. However, their widespread use has led to the emergence of bacterial pathogens with increased

Communicated by Erko Stackebrandt.

Mark Shepherd

M.Shepherd@kent.ac.uk

1 School of Biosciences, RAPID Group, University of Kent, Canterbury CT2 7NJ, UK

2 Clinical Microbiology Service, East Kent Hospitals University NHS Foundation Trust, William Harvey Hospital, Ashford, Kent TN24 0LZ, UK

3 Laboratoire National de Santé 1, Rue Louis Rech, L-3555 Dudelange, Luxembourg

4 School of Science, Engineering \& Environment, University of Salford, Lancashire M5 4WT, UK resistance to antimicrobial agents, leading to an increase in mortality and morbidity. Recently, the emergence of multidrug-resistance (i.e. to three or more antibiotic classes) in Gram-negative pathogens has become a cause for concern, with several Gram-negative bacteria, including E. coli, found to be NDM-1 $\beta$-lactamase-producing (Walsh et al. 2011). These strains often carry many other resistant determinants resulting in extreme multidrug-resistant phenotypes (Yong et al. 2009). Of particular concern is bacteraemia caused by E. coli, where there are approximately 40,000 annual cases in England. Over one-third of these are caused by drug-resistant strains causing close to 5,000 deaths each year (Public Health England). An over-represented clonal group is E. coli ST131, a uropathogen first identified in 2008 (Nicolas-Chanoine et al. 2008) that is now globally disseminated in both hospital and community settings and has a high incidence of CTX-M-15 extended-spectrum $\beta$-lactamase (ESBL)-producing isolates (Croxall et al. 2011; NicolasChanoine et al. 2014). Given the apparent ease with which 
multidrug-resistant $E$. coli are able to spread throughout the human population, it is important to understand elements of the host environment and bacterial physiology that could potentially subvert the efficacy of antibiotics. Indeed, the ability of a bacterium to respire has a profound impact upon antibiotic function and is, therefore, a major focus of the current study. A recent study has shown that the abolition of aerobic respiration (reviewed in (Stokes et al. 2019)), dramatically reduces the toxic effects of bactericidal antibiotics (Lobritz et al. 2015). It, therefore, seems reasonable that a potent respiratory inhibitor such as a nitric oxide (NO), which is produced by the host immune system during infection, would also impair antibiotic efficacy.

It is well-known that bactericidal antibiotics can induce cell death via activation of pathways that result in oxidative damage via elevation of aerobic respiratory rates (Dwyer et al. 2007, 2014; Foti et al. 2012), with superoxide production implicated as a key intermediate: superoxide causes destabilisation of iron-sulphur complexes (Imlay and Fridovich 1991; Keyer and Imlay 1996) which causes iron dysregulation and, more importantly, leaching of ferrous iron. Subsequent reactions of leached ferrous iron reduce hydrogen peroxide to toxic hydroxyl radicals via the Fenton reaction (Imlay et al. 1988). Although different bactericidal antibiotics have different modes of action, Kohanski and colleagues (Kohanski et al. 2007, 2010) identified that most are able to induce the formation of hydroxyl radicals which is thought to be linked to the aerobic respiratory chain. However, for aminoglycosides, there is significant evidence to support a link between the proton motive force and antibiotic toxicity that is independent of ROS formation: uptake of positively charged aminoglycosides is linked to the electrical component of the proton motive force, as transport into the cell is thermodynamically favourable as the charge component of the PMF will be dissipated upon antibiotic entry (Taber et al. 1987; Farha et al. 2018).

Aside from the potential role in disrupting the potency of antibiotics, the primary function of NO in the immune response is to act as a toxic antimicrobial to combat infection. Cellular targets for NO include thiols, metal centres, haem cofactors, and cysteine residues of proteins that are susceptible to $S$-nitrosylation (Fang 1997; Keszler et al. 2010). Bacteria have encountered NO in the gut and at the local site of infection for millennia, and have predictably evolved a variety of mechanisms to circumvent this toxicity (Shepherd et al. 2016), including detoxification systems to convert NO to less toxic substances, NO-tolerant respiratory oxidases, and mechanisms to repair damaged iron sulphur clusters. Hence, an assumption made in the current study is that bacterial exposure to NO and the evolution of resistance to this toxic molecule pre-dates the widespread exposure to antibiotics and the resultant emergence of antibiotic resistance. Given that loss of aerobic respiratory complexes had previously been shown to diminish the efficacy of various antibiotics (Lobritz et al. 2015), it was anticipated that the respiratory inhibitor NO would also diminish antibiotic susceptibility. If this could be demonstrated, we hypothesised that hyper-sensitivity to NO may have arisen in bacterial pathogens as a mechanism to promote the acquisition of antibiotic-resistance through enabling cells to persist in the presence of toxic levels of antibiotic (Fig. 1). Hence, the current work sought to investigate the relationship between NO tolerance/sensitivity and carriage of antibiotic resistance genes and to characterise the impact of NO upon the efficacy of conventional antibiotics against $E$. coli clinical isolates.

\section{Materials and methods}

\section{Bacterial isolates}

Fifty E. coli blood culture isolates were collected from East Kent Hospitals University NHS Foundation Trust. Approval was sought from the Research Ethics Council in advance (ref: 12/SC/0673) for this fully-blinded study. $E$. coli CFT073 is a highly invasive UPEC strain and a causative agent of pyelonephritis (Mobley et al. 1990; Welch et al. 2002), E. coli 83972 is asymptomatic bacteriuria (ABU) strain capable of out-competing UPEC strains (Roos et al. 2006), and EC958 is a well-characterised O25: H4-ST131 multidrug-resistant urosepsis-causing strain (Totsika et al. 2013; Forde et al. 2014). EC958 Cmcassette insertional mutant strains norVW and $h m p$ have been described previously (Shepherd et al. 2016).

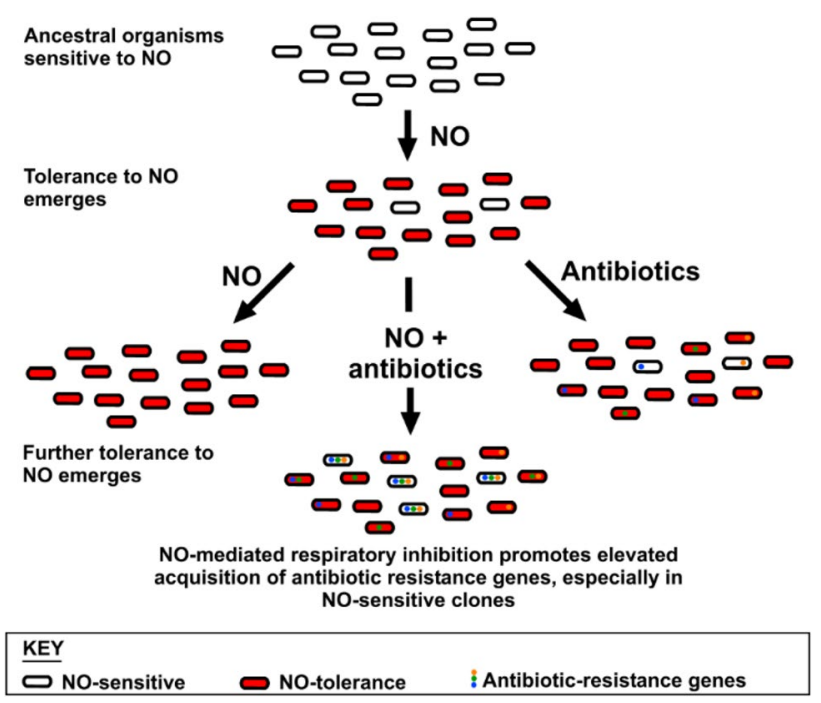

Fig. 1 Initial hypotheses for the co-evolution of NO sensitivity and antibiotic resistance gene carriage 


\section{Genomics analyses}

Multilocus sequence typing (MLST) was performed using the Achtman approach as previously described (Maiden et al. 1998; Clark et al. 2012). A minimum spanning tree was created based on the MLST data using BioNumerics version 7.5 created by Applied Maths NV. Bacterial genomes were sequenced using an Illumina MiSeq instrument, and reads were assembled de novo using SPAdes Genome Assembler (Bankevich et al. 2012), after which the genomes were reordered with the software Mauve (v2.4.0) (Darling et al. 2004) and using the well-characterised genome E. coli MG1655 (NC_000913.3) as reference. The newly re-ordered genomes were annotated with Prokka (v1.12) (Seemann 2014). Pan-genome analysis was accomplished with Roary (Page et al. 2015), and the core genome obtained was used as input in FastTree (Price et al. 2009) to create a phylogenetic tree of all the isolates of the Kent Collection, and edited with iTOL v3 (Letunic and Bork 2016). Genomes were mined for virulence genes using ABRicate (v0.8) (https ://github.com/tseemann/abricate) to search the VFDB database (Chen et al. 2005). Protein sequences were aligned with T- Coffee (Notredame et al. 2000; Di Tommaso et al. 2011) and the values for protein identity and similarity of each pairwise alignment were extracted with BioEdit v7.2.5. For phylogenetic group determination, the Clermont method was used (Clermont et al. 2013, 2015). Briefly, the genomic sequence of each isolate was concatenated in FASTA format and used for in silico PCR using Unipro UGENE platform (Okonechnikov et al. 2012) with the primer sequences used by Clermont et al. (Clermont et al. 2013). To predict antibiotic susceptibility using genomic data, genomes were mined for antibiotic resistance genes with ABRicate (https ://github.com/tseemann/abricate) to search the ResFinder 2.1 database (Zankari et al. 2012). For in silico detection of aminoglycoside resistance, the protein sequences of the gyrase subunit A for all genomes were aligned with T-Coffee (Notredame et al. 2000; Di Tommaso et al. 2011) and chromosomal mutations known to elicit resistance (Weigel et al. 1998; McArthur et al. 2013; Jia et al. 2017) were manually detected.

\section{Antimicrobial susceptibility assays}

The British Society for Antimicrobial Chemotherapy (BSAC) method for antibiotic susceptibility testing was used to prepare standardised inoculum (Andrews 2001, 2002) and for breakpoint values (i.e. antimicrobial threshold concentrations that define resistance/susceptibility). Antimicrobial susceptibility profiles of $E$. coli clinical isolates were obtained for amoxicillin (AMX) (Sigma), cefotaxime (CTX) (Sigma), chloramphenicol (CAP) (ThermoFisher), ciprofloxacin (CIP) (Fluka), meropenem (MEM) (Sigma), nitrofurantoin (NIT) (Sigma), and trimethoprim (TMP) (Sigma) using the disc diffusion method. Due to poor agar diffusion, the susceptibility of the clinical isolates to polymyxin E (PME) (Sigma) was determined using the minimum inhibitory concentration (MIC) method.

\section{Gentamicin dose response assays}

Cultures of EC958 wild type were grown to stationary phase overnight in LB at $37^{\circ} \mathrm{C} .10 \mathrm{~mL}$ volumes of $\mathrm{M} 9$ media $(16 \mathrm{~g} / \mathrm{L}$ $\mathrm{Na}_{2} \mathrm{HPO}_{4} \cdot 2 \mathrm{H}_{2} \mathrm{O}, 3 \mathrm{~g} / \mathrm{L} \mathrm{KH}_{2} \mathrm{PO}_{4}, 0.5 \mathrm{~g} / \mathrm{L} \mathrm{NaCl}, 1 \mathrm{~g} / \mathrm{L} \mathrm{NH}_{4} \mathrm{Cl}$, $0.24 \mathrm{~g} / \mathrm{L} \mathrm{MgSO}_{4}, 0.01 \mathrm{~g} / \mathrm{L} \mathrm{CaCl}_{2}$, and $4 \mathrm{~g} / \mathrm{L}$ glucose) supplemented with $0.1 \%$ casamino acids (w/v) were prepared in $50 \mathrm{~mL}$ conical flasks and inoculated with $1 \%(\mathrm{v} / \mathrm{v})$ of the stationary phase cultures, and incubated at $37^{\circ} \mathrm{C}, 180 \mathrm{rpm}$ until mid-exponential phase. From these cultures, suspensions of $10^{8} \mathrm{CFU} / \mathrm{mL}$ were prepared in fresh M9 media supplemented with $0.1 \%$ casamino acids and exposed to GSNO $(0$ or $15 \mathrm{mM})$ or NOC-12 (0 or $1 \mathrm{mM})$ for $30 \mathrm{~min}$ at $37{ }^{\circ} \mathrm{C}$, prior to incubation with different concentrations of gentamicin for $90 \mathrm{~min}$. Serial dilutions were performed in $1 \times$ PBS and cells were plated in triplicate on LB-agar plates. Colony counts were performed after overnight incubation at $37^{\circ} \mathrm{C}$. Two biological repeats were performed for each condition, with three technical repeats performed for each biological repeat (i.e. 6 in total).

\section{Nitric oxide susceptibility assays}

Nitric oxide susceptibility was evaluated using a well diffusion assay. Briefly, cultures at mid-exponential growth were prepared in M9 minimal medium supplemented with $0.1 \%$ casamino acids, using a $1 \%$ inoculum of stationary phase aerobic culture prepared in LB medium at $37{ }^{\circ} \mathrm{C}$. When mid$\log$ phase was reached, cells were plated on M9 minimal agar medium using the pour plate technique. Six $6 \mathrm{~mm}$ diameterwells were cut in the agar gel and $80 \mu \mathrm{L}$ of $80 \mathrm{mM} \mathrm{S}$-Nitrosoglutathione (GSNO), synthesised and quantified as previously described (Hart 1985), was added to each. Plates were incubated at $37{ }^{\circ} \mathrm{C}$ under aerobic, microaerobic ( $2 \%$ oxygen) or anaerobic conditions. For anaerobic growth, $50 \mathrm{mM}$ fumarate was included in the agar. For microaerobic growth, oxygen levels were controlled using a Ruskinn Invivo 300 Hypoxia Workstation and for anaerobic growth, plates were incubated in anaerobic jars with an anaerobic gas pouch (Oxoid) and oxygen indicator (Oxoid). Zones of inhibition were measured after $16 \mathrm{~h}$ incubation, and data were analysed using a twotailed unpaired Student's $t$-test. 


\section{Results}

\section{Nitric oxide diminishes antibiotic efficacy}

It has previously been demonstrated that NO protects $\mathrm{Sal}$ monella enterica, Pseudomonas aeruginosa and Staphylococcus aureus from aminoglycosides by blocking the energy-dependent phases of drug uptake (McCollister et al. 2011), although no detailed analyses were undertaken to measure the impact upon antibiotic $\mathrm{IC}_{50}$ values, and no work was undertaken on pathogenic $E$. coli. Hence, a gentamicin dose response killing experiment was conducted with the multidrug-resistant $E$. coli $\mathrm{O} 25 \mathrm{~b}: \mathrm{H} 4-$ ST131 (EC958) strain (Totsika et al. 2013; Forde et al. 2014) using the NO-donor GSNO. Pre-exposure of an exponentially-growing culture of EC958 to $15 \mathrm{mM}$ GSNO for $30 \mathrm{~min}$, prior to the addition of increasing gentamicin concentrations for $90 \mathrm{~min}$, led to a dramatic increase in bacterial survival (Fig. 2a) with the $\mathrm{IC}_{50}$ increasing approximately tenfold for cells pre-exposed to GSNO. To confirm that this effect was due to the presence of $\mathrm{NO}$, a similar experiment was performed with $1 \mathrm{mM}$ NOC-12 in place of GSNO (Fig. 2b), which confirmed the same pattern with a slightly more pronounced effect: pre-treatment with NOC-12 resulted in a 16-fold increase in the $\mathrm{IC}_{50}$ of gentamicin. A GSNO-mediated increase in $\mathrm{IC}_{50}$ was also observed under anaerobic conditions (Fig. S1), and it was also noted that a switch to anaerobiosis elicited a $>$ tenfold increase in the $\mathrm{IC}_{50}$ for gentamicin in the absence of GSNO.

\section{No detectable correlation exists between antibiotic-resistance gene carriage and nitric oxide susceptibility in E. coli clinical isolates}

To investigate the potential relationship between antibiotic-resistance gene carriage and NO-mediated respiratory inhibition, it was necessary to perform genomic and phenotypic analysis of $E$. coli clinical isolates. Fifty $E$. coli bacteraemia isolates were collected from the Clinical Microbiology Service at The William Harvey Hospital (Ashford, Kent) in a double-blinded study, and will be herein referred to as the Kent Collection (KC). MLST analysis performed on $\mathrm{KC}$ isolates identified a total of 23 unique sequence types (STs), with the most prevalent STs ST73, ST69, and ST131, accounting for $18 \%, 14 \%$, and $12 \%$ of the collection, respectively (Fig. S2). Isolates were sequenced using the Illumina MiSeq platform, and phylogenetic analysis with Roary (Page et al. 2015) was used to perform phylogroup clustering based on core genomes
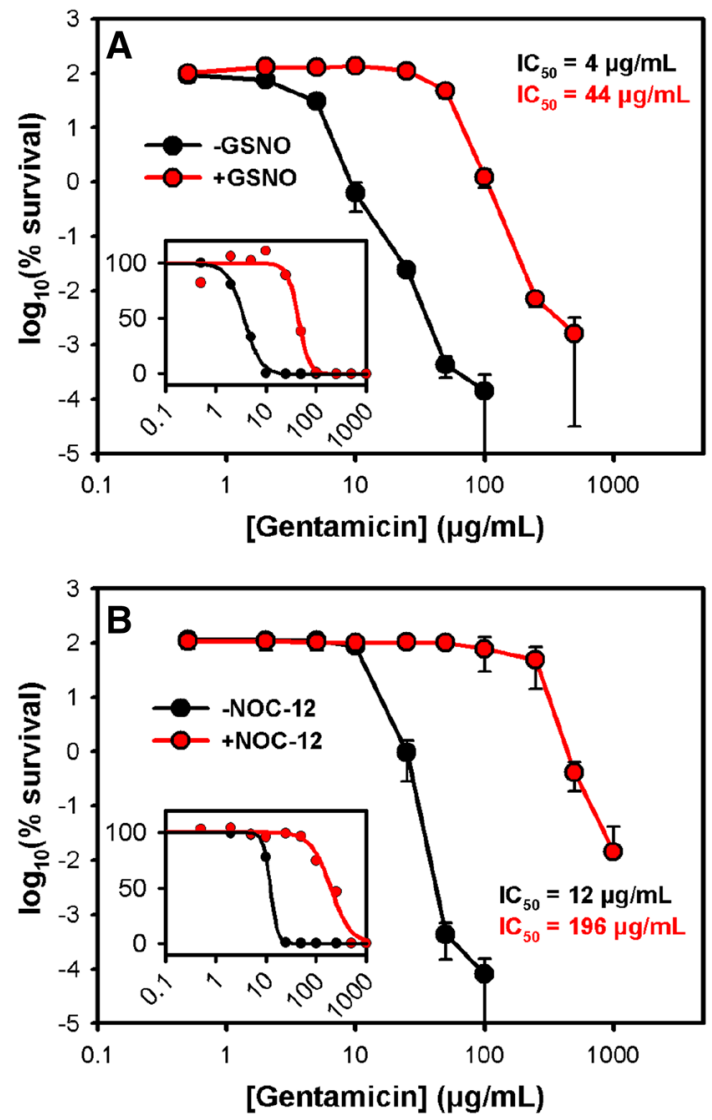

Fig. 2 Nitric oxide releasers abrogate the lethality of gentamicin. Suspensions of $10^{8}$ cells $/ \mathrm{mL}$ of E. coli EC958 in M9 minimal media supplemented with $0.1 \%$ casamino acids were exposed to $15 \mathrm{mM}$ GSNO (a) and $1 \mathrm{mM}$ NOC-12 (b) for $30 \mathrm{~min}$, followed by incubation with different concentrations of gentamicin for $90 \mathrm{~min}$. Serial dilutions were performed in PBS and plated on $\mathrm{LB}$-agar. $\mathrm{CFU} / \mathrm{mL}$ values were determined after overnight incubation at $37^{\circ} \mathrm{C}$. Data were fitted to a four parameter Hill equation for calculation of $\mathrm{IC}_{50}$ values, and magnitude changes were normalised to $100 \%$ for display on a linear $\mathrm{y}$-axis (insets). Values shown represent mean $\pm \mathrm{SD}$ from two biological repeats, each comprising three technical repeats

(Clermont et al. 2013, 2015) (Fig. 3a). To characterise antibiotic susceptibility and resistance gene carriage in this collection, a combination of in silico and laboratory techniques were employed. This dual approach was undertaken as in silico techniques could be used to rapidly screen all isolates for resistance to many classes of antibiotic, and then the more labour intensive experimental laboratory approach was undertaken on a subset of the antibiotics to validate the in silico results. These analyses revealed that $14 \%$ of the collection exhibited multidrug resistance (i.e. resistant to three or more antibiotic classes), with three such isolates belonging to the ST131 clonal group (Fig. 3a, outer ring). Furthermore, $6 \%$ were CTX-M-15 ESBL-producing isolates, and susceptibility to amoxicillin, trimethoprim, and ciprofloxacin was found in 


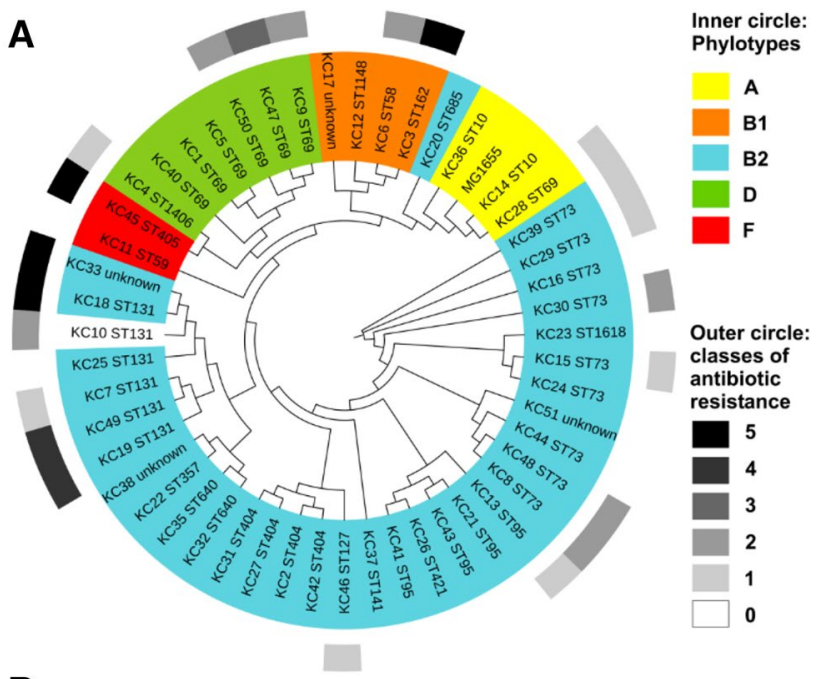

B

\begin{tabular}{|c|c|c|c|c|c|c|c|c|c|c|c|c|}
\hline \multirow[b]{2}{*}{ ST } & \multicolumn{12}{|c|}{$\%$ Antibiotic Resistance (in silico data) } \\
\hline & AMX & CTX & CAP & CIP & GEN & MEM & NIT & TMP & $\mathrm{PM}$ & ML & TE & SUL \\
\hline 131 & 83 & 17 & 0 & 83 & 33 & 0 & 0 & 50 & 0 & 33 & 17 & 67 \\
\hline & 43 & 0 & 14 & 14 & 0 & 0 & 0 & 43 & 0 & 14 & 29 & 43 \\
\hline & 67 & 0 & 0 & 0 & 0 & 0 & 0 & 33 & 0 & 0 & 22 & 67 \\
\hline & 25 & 7 & 6 & 13 & 7 & 0 & 0 & 21 & 0 & 7 & 19 & 25 \\
\hline
\end{tabular}

\begin{tabular}{|c|c|c|c|c|c|c|c|c|c|c|}
\hline \multirow[b]{2}{*}{ ST } & \multicolumn{9}{|c|}{ \% Antibiotic Resistance (Experimental data) } & \multirow{2}{*}{$0 \%$} \\
\hline & AMX & CTX & CAP & CIP & GEN & MEM & NIT & TMF & PME & \\
\hline ST131 & 83 & 17 & 0 & 67 & 50 & 0 & 0 & 50 & 0 & \\
\hline ST69 & 67 & 0 & 0 & 17 & 0 & 0 & 0 & 50 & 0 & \\
\hline ST73 & 67 & 0 & 0 & 0 & 0 & 0 & 0 & 33 & 0 & \\
\hline Others & 21 & 7 & 7 & 11 & 7 & 0 & 4 & 18 & 0 & \\
\hline
\end{tabular}

Fig. 3 Antibiotic susceptibilities of E. coli phylogroups and sequence types. a Phylogenetic relationships of 50 E. coli clinical isolates were inferred using the core genome gene sequences of all isolates (compared to E. coli MG1655) obtained by pan-genome analysis with Roary. Phylogroups for each isolate were determined using in silico $\mathrm{PCR}$, and each colour represents one phylogroup in the tree (inner circle). b Antibiotic resistance profiles were predicted using bioinformatics approaches and were also experimentally screened for antibiotic resistance using disc susceptibility testing (Andrews 2001, 2002) (the total number of antibiotic classes that each isolate is resistant to, based on disc susceptibility, is shown in the outer ring of (a)). ' $\%$ antibiotic resistance' is defined as the $\%$ of isolates within a sequence type that are resistant to a particular antibiotic. $S T$ sequence type, $A M X$ amoxicillin, $C T X$ cefotaxime, $C A P$ chloramphenicol; $C I P$ ciprofloxacin, Gen gentamicin, MEM meropenem, NIT nitrofurantoin, $T M P$ trimethoprim, $P M E$ polymyxin E, $M L S$ macrolides, TET tetracycline, $S U L$ sulfanilamides

$42 \%, 28 \%$, and $16 \%$ of the isolates, respectively. Resistance data for the most prevalent STs, which are also the clonal groups with the highest degree of antibiotic resistance (i.e. ST131, ST69, ST73), are shown in Fig. 3b.

To investigate potential correlations between antibiotic resistance and tolerance to $\mathrm{NO}$, isolates that exhibited the highest degree of antibiotic resistance for each ST were selected (Fig. 4a), and susceptibility to the NO-releasing molecule GSNO was measured both in the presence (aerobic and microaerobic) and absence (anaerobic) of oxygen (Fig. 4b). Well-characterised E. coli clinical isolates EC958
(Totsika et al. 2013; Forde et al. 2014), CFT073 (Mobley et al. 1990; Welch et al. 2002) and 83,972 (Roos et al. 2006) were also analysed as control samples and known NO-sensitive mutant strains were confirmed to have larger zones of inhibition to validate the technique (Fig. S3). Out of the 13 strains tested, none of the isolates displayed a significant increase in NO tolerance compared to the control strains (Student's unpaired two-tailed $t$-tests), and only one isolate (KC45) exhibited significantly higher susceptibility to NO than all other strains $(P$-value $\leq 0.001)$ under both aerobic and microaerobic conditions tested. However, further assessment of $\mathrm{NO}$-sensitivity using the alternative NO-releasing compound NOC-12 revealed no sensitivity (Fig. S4), suggesting that this $\mathrm{KC} 45$ isolate is sensitive to a GSNO breakdown product other than NO. For this reason, the KC45 strain was omitted from the correlation analyses between antibiotic resistance and GSNO sensitivity for the different oxygen conditions, where no significant relationship was observed (Fig. 4c). To investigate potential correlations between antibiotic resistance and virulence gene carriage, genomic sequences were mined for virulence genes (Fig. 5a), although no significant correlation was found between the degree of antibiotic resistance and virulence profile from the Pearson Correlation Coefficient (Fig. 5b).

\section{Discussion}

The antimicrobial properties of nitric oxide are well-known, and it has long been considered a potential alternative to antibiotics or for use in combination with antibiotics. However, given the anticipated potent inhibition of respiration in the presence of $\mathrm{NO}$ in aerobically-growing planktonic $E$. coli cells, it was hypothesised that NO would enhance tolerance to antibiotics (Fig. 1). Indeed, pre-exposure of $E$. coli EC958 with the NO-donors GSNO or NOC-12 prior to treatment with gentamicin dramatically increased (i.e. $>$ tenfold increase in $\mathrm{IC}_{50}$ ) bacterial tolerance to this antibiotic (Fig. 2). In addition, GSNO was shown to offer significant protection to gentamicin in anaerobically-grown cells (Fig. S1), which suggests that there are cellular targets for GSNO other than respiratory oxidases that can influence tolerance to aminoglycosides. Interestingly, the $\mathrm{IC}_{50}$ for gentamicin increased by $>$ tenfold when cells were grown anaerobically without GSNO, highlighting the dramatic influence that growth conditions can have upon antibiotic susceptibility. Given that NO is produced by the host immune system during infection, these data should change the way that antibiotic susceptibility is studied both during infection and in microbiological culture media and it would be particularly interesting to re-assess $\mathrm{IC}_{50}$ values for all antibiotics and all organisms via the BSAC method in the presence of NO for cells grown both aerobically and anaerobically. 
Fig. 4 Antibiotic-resistant $E$. coli clinical isolates are not resistant/sensitive to NO. a Antibiotic resistance profiles of the most resistant isolates in the KC collection compared to MG1655, EC958, CFT073, and $A B U$ strain 83972. AMX Amoxicillin, CTX Cefotaxime, $C A P$ Chloramphenicol, $C I P$ Ciprofloxacin, MEM Meropenem, NIT Nitrofurantoin, TMP Trimethoprim, $P M E$ Polymyxin E; (-) Sensitive; (+) Resistant. ${ }^{1}$ N/A Not Applicable. b NO susceptibility of antimicrobialresistant isolates representative of STs, under different oxygen conditions. Values shown are the mean \pm SD from three experiments. $(*$ Student's $t$-test unpaired two-tail $P$-value $<0.05)$. c Linear regression and correlation between GSNO susceptibility and antibiotic resistance. Correlation between GSNO susceptibility and the degree of antibiotic resistance (reflected by the number of antibiotics to which each isolate was experimentally resistant to) was calculated using the Pearson $r$ for all strains in panels A and B except KC45 (Pearson $r=0.08,0.04$ and 0.19 for aerobic, microaerobic, and anaerobic conditions respectively. $P$-values of 0.79 , 0.91 , and 0.56 were obtained for aerobic, microaerobic, and anaerobic conditions, respectively). Linear relation between the two variables was assessed with linear regression, with $R^{2}$ values of $0.007,0.001$, and 0.035 for aerobic, microaerobic, and anaerobic conditions, respectively)
A

\begin{tabular}{lccccccccccc}
\hline Strain & ST & Notes & AMX & CTX & CAP & CIP & GEN & MEM & NIT & TMP & PME \\
\hline MG1655 & N/A & K-12 & - & - & - & - & - & - & - & - & - \\
EC958 & ST131 & UPEC & + & + & - & + & - & - & - & + & - \\
CFT073 & ST73 & UPEC & - & - & - & - & - & - & - & - & - \\
\hline 83972 & N/A & ABU & - & - & - & - & - & - & - & - & - \\
KC3 & ST162 & & + & - & + & + & - & - & + & + & - \\
KC4 & ST1406 & & + & - & - & - & - & - & - & - & - \\
KC6 & ST58 & & + & - & - & - & - & - & - & + & - \\
KC13 & ST95 & UPEC & - & - & - & - & - & - & - & + & - \\
KC30 & ST73 & UPEC & + & - & - & - & - & - & - & + & - \\
KC33 & Unknown & & + & + & - & + & + & - & - & + & - \\
KC45 & ST405 & UPEC & + & + & - & + & + & - & - & + & - \\
KC46 & ST127 & UPEC & + & - & - & - & - & - & - & - & - \\
KC47 & ST69 & UPEC & + & - & - & + & - & - & - & + & - \\
\hline
\end{tabular}

B
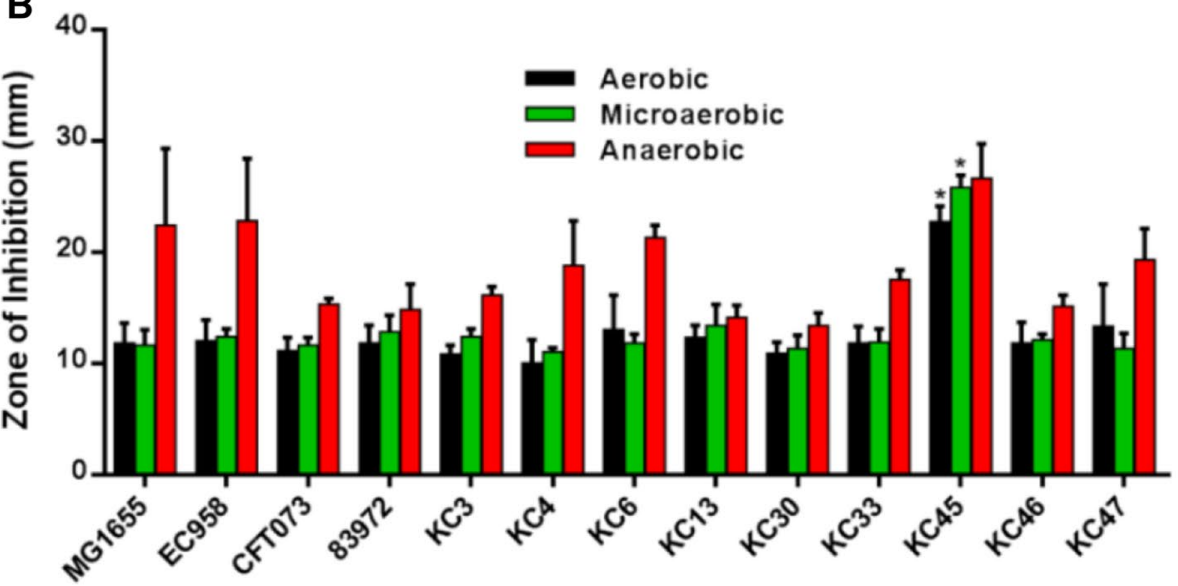

C

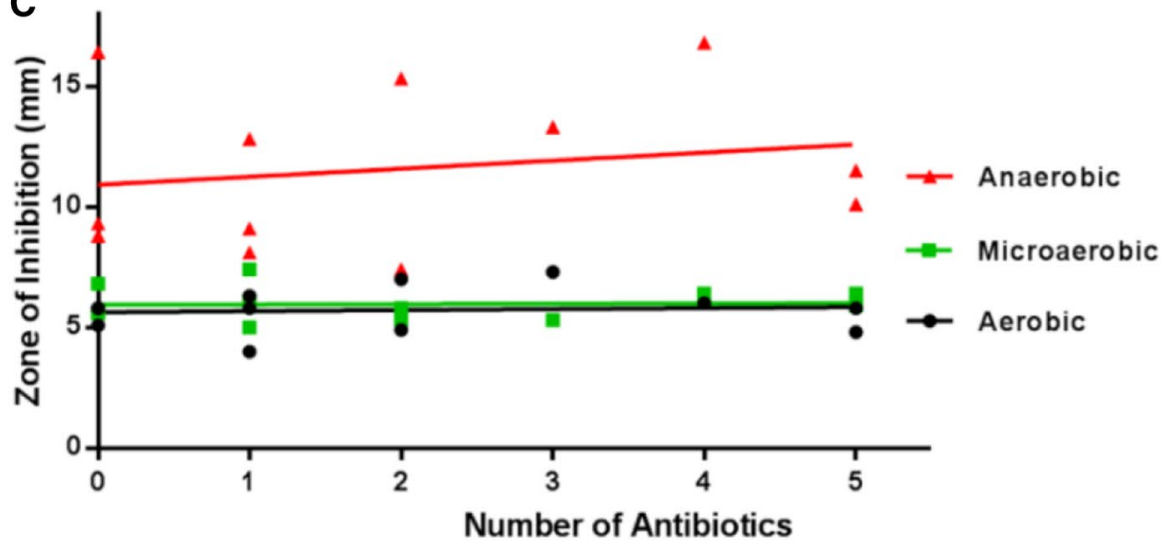

Given that both $\mathrm{NO}$ and antibiotics are encountered during infection, and that susceptibility to NO-mediated growth inhibition can prolong exposure of bacteria to antibiotics, it was hypothesised that hypersensitivity to NO could serve as an evolutionary driver for the acquisition of antibiotic resistance genes (Fig. 1). To investigate this, genomic characterisation of $50 \mathrm{E}$. coli bacteraemia isolates was undertaken and revealed the three most common STs to be ST73, ST69, and ST131 (Fig. 3), which have previously been shown to be comprised of multidrug-resistant strains (Croxall et al. 2011). In this collection, the ST131 isolates contained a greater incidence of antibiotic resistance compared to ST73, which is consistent with previous studies (Johnson et al. 2010; Forde et al. 2014; NicolasChanoine et al. 2014b). Deletion of all three respiratory oxidase complexes in a non-pathogenic strain of $E$. coli has previously been shown to confer tolerance to antibiotics (Lobritz et al. 2015), so it was hypothesised that some bacterial isolates may have evolved sensitivity to the respiratory inhibitor NO, which could prolong survival in 

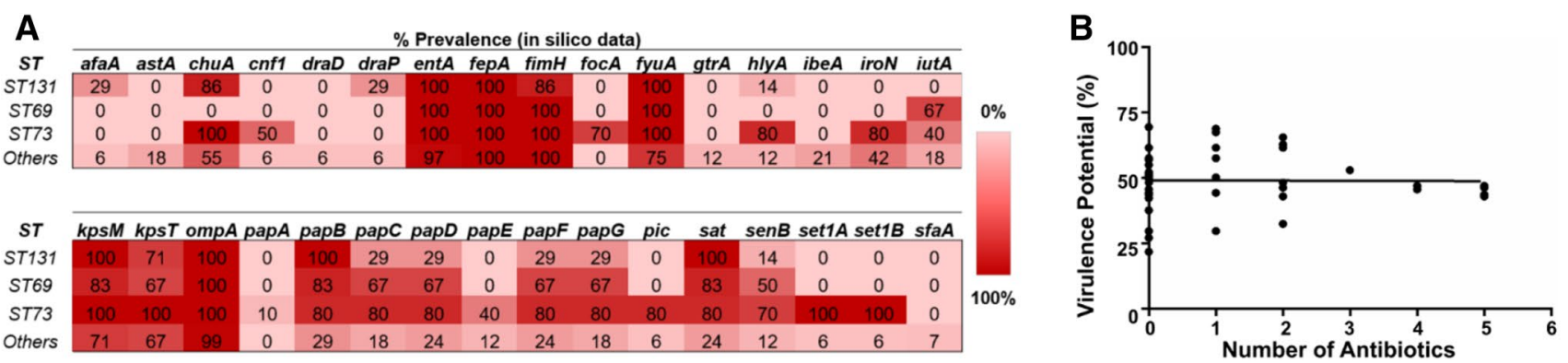

Fig. 5 Virulence gene profiles of E. coli bacteraemia isolates: no correlation with antibiotic resistance. a 50 clinical isolates were sequenced and virulence genes were identified using bioinformatics approaches. A selection of genes is shown for the most abundant sequence types in the collection. b The correlation between the virulence potential and the degree of antibiotic resistance (reflected by the number of different antibiotic classes to which each isolate was experimentally resistant to) was investigated by calculating the Pearson Correlation Coefficient (Pearson $r=-0.0097 ; P$-value $=0.95$ ). Virulence potential is calculated as the $\%$ of the 151 virulence genes identified that are carried in a given isolate. A relationship between the two variables was assessed using linear regression $\left(R^{2}=9.4 \times 10^{-5}\right) . S T$ sequence type

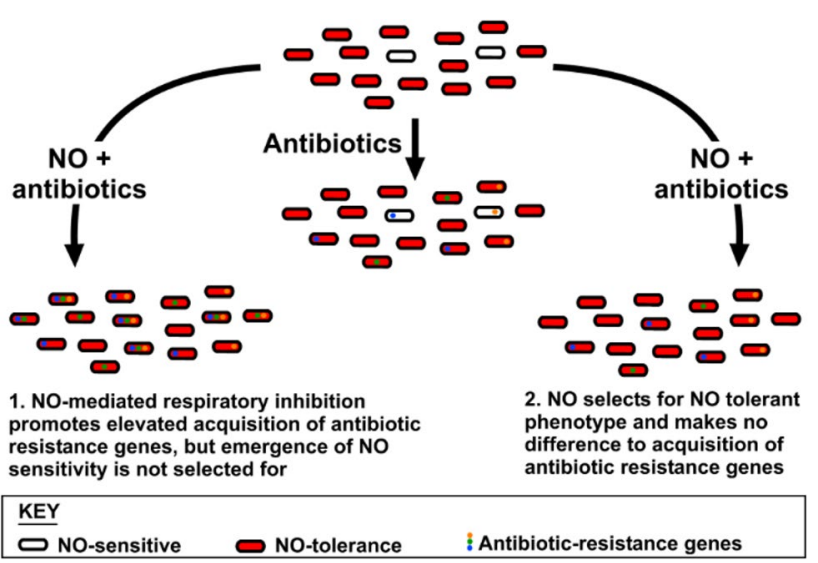

Fig. 6 Alternative explanations to explain the relationship between NO tolerance and antibiotic resistance gene carriage. Two alternative explanations are provided to explain the absence lack of correlation observed in the current study between NO sensitivity and antibiotic resistance gene carriage

Previous work has demonstrated an inverse correlation between antibiotic resistance and virulence in $S$. aureus (Sapri et al. 2013) and there are many reports of resistance mechanisms affecting virulence (Beceiro et al. 2013), so it was of interest to screen this collection for correlations of this sort. However, no relationship between virulence and antibiotic resistance was observed in the $\mathrm{KC}$ collection studied herein (Fig. 5).

Given the direct toxic effects of NO upon bacterial cells (Fig. 2) and the ability of NO to disperse biofilms, NOreleasing compounds are potentially useful to treat bacterial infections where conventional antibiotics are no longer effective. Indeed, a combination of NO releasers and ciprofloxacin has previously been shown to be effective upon acquisition of antibiotic resistance genes. 
against $E$. coli and $P$. aeruginosa biofilms (de la FuenteNúñez et al. 2013; Reffuveille et al. 2015). However, the current data show that NO has the opposite effect for gentamicin and planktonic E. coli cells, potentially due to NOmediated respiratory inhibition diminishing the efficacy of the aminoglycoside gentamicin. More broadly speaking, diminished metabolic activity is becoming widely accepted to have a profound impact upon antibiotic susceptibility in general (Stokes et al. 2019), so the impairment of metabolic activity beyond respiratory metabolism caused by NO exposure (e.g. through destruction of iron-sulphur clusters and protein $S$-nitrosation) is likely to reduce the efficacy of a variety of antibiotic classes. While no strains with increased NO sensitivity were detected herein, no strains with increased NO tolerance were present either, suggesting that the entire collection would be resistant to gentamicin (and other antibiotics) in the presence of NO. With the potent effects that NO has upon antibiotic efficacy in clinical isolates in mind, it is clearly important to fully understand the combined effects of antibiotic treatments and nitrosative stresses, especially when devising new strategies to combat multidrug-resistant strains.

Supplementary Information The online version contains supplementary material available at https://doi.org/10.1007/s00203-021-02245-2.

Acknowledgements We thank Prof. Mark Schembri (University of Queensland) for sharing well-characterised E. coli isolates. We are grateful for the use of the MRC CLIMB microbial informatics infrastructure funded under Grant Reference MR/L015080/1. We acknowledge genome sequencing provided by MicrobesNG (http://www.micro besng.uk), which is supported by the BBSRC (grant number BB/ L024209/1). We also acknowledge Prof. Alan McNally (University of Birmingham) and Dr. Fahad Alhashash (Nottingham Trent University) for performing the MLST experiment.

Authors' contributions CAR performed the majority of experiments and wrote the paper, LAR, LGH, CMW and AMW performed the gentamicin dose response work, TIM contributed to the genome assembly and annotation work, GKR contributed to microbiological experimental design, FAM supplied clinical isolates and contributed to microbiological experimental design, IBG supervised the genome sequencing/ assembly/annotation work, and MS was responsible for experimental design and wrote the paper.

Funding This work was supported by a Strategic Research Development Fund (KentHealth) and a KentHealth PhD studentship (University of Kent). IBG was supported by the University of Salford Research Support fund.

\section{Compliance with ethical standards}

Conflict of Interest The authors declare that there is no conflict of interest.
Ethical approval For collection of E. coli bacteraemia isolates, approval was sought from the Research Ethics Council in advance (ref: $12 / \mathrm{SC} / 0673$ ) for this fully-blinded study.

Open Access This article is licensed under a Creative Commons Attribution 4.0 International License, which permits use, sharing, adaptation, distribution and reproduction in any medium or format, as long as you give appropriate credit to the original author(s) and the source, provide a link to the Creative Commons licence, and indicate if changes were made. The images or other third party material in this article are included in the article's Creative Commons licence, unless indicated otherwise in a credit line to the material. If material is not included in the article's Creative Commons licence and your intended use is not permitted by statutory regulation or exceeds the permitted use, you will need to obtain permission directly from the copyright holder. To view a copy of this licence, visit http://creativecommons.org/licenses/by/4.0/.

\section{References}

Andrews JM (2001) Determination of minimum inhibitory concentrations. J Antimicrob Chemother 48:5-16. https://doi.org/10.1093/ jac/48.suppl_1.5

Andrews JM (2002) BSAC standardized disc susceptibility testing method. J Antimicrob Chemother 48: Suppl. S1, 43-57. https:// doi.org/10.1093/jac/dkf084

Bankevich A, Nurk S, Antipov D et al (2012) SPAdes: a new genome assembly algorithm and its applications to single-cell sequencing. J Comput Biol 19:455-477. https://doi.org/10.1089/ cmb.2012.0021

Beceiro A, Tomas M, Bou G (2013) Antimicrobial resistance and virulence: a successful or deleterious association in the bacterial world? Clin Microbiol Rev 26:185-230. https://doi.org/10.1128/ CMR.00059-12

Chen L, Yang J, Yu J et al (2005) VFDB: a reference database for bacterial virulence factors. Nucleic Acids Res 33:D325-D328. https://doi.org/10.1093/nar/gki008

Clark G, Paszkiewicz K, Hale J et al (2012) Genomic analysis uncovers a phenotypically diverse but genetically homogeneous Escherichia coli ST131 clone circulating in unrelated urinary tract infections. J Antimicrob Chemother 67:868-877. https://doi.org/10.1093/ $\mathrm{jac} / \mathrm{dkr} 585$

Clermont O, Christenson JK, Denamur E, Gordon DM (2013) The Clermont Escherichia coli phylo-typing method revisited: improvement of specificity and detection of new phylo-groups. Environ Microbiol Rep 5:58-65. https://doi.org/10.1111/1758-2229.12019

Clermont O, Gordon D, Denamur E (2015) Guide to the various phylogenetic classification schemes for Escherichia coli and the correspondence among schemes. Microbiology 161:980-988. https ://doi.org/10.1099/mic.0.000063

Croxall G, Hale J, Weston V et al (2011) Molecular epidemiology of extraintestinal pathogenic Escherichia coli isolates from a regional cohort of elderly patients highlights the prevalence of ST131 strains with increased antimicrobial resistance in both community and hospital care setting. J Antimicrob Chemother 66:2501-2508. https://doi.org/10.1093/jac/dkr349

Darling ACE, Mau B, Blattner FR, Perna NT (2004) Mauve: multiple alignment of conserved genomic sequence with rearrangements. Genome Res 14:1394-1403. https://doi.org/10.1101/gr.2289704

de la Fuente-Núñez C, Reffuveille F, Fairfull-Smith KE, Hancock REW (2013) Effect of nitroxides on swarming motility and biofilm formation, multicellular behaviors in Pseudomonas aeruginosa. 
Antimicrob Agents Chemother 57:4877-4881. https://doi. org/10.1128/AAC.01381-13

Di Tommaso P, Moretti S, Xenarios I et al (2011) T-Coffee: a web server for the multiple sequence alignment of protein and RNA sequences using structural information and homology extension. Nucleic Acids Res 39:W13-W17. https://doi.org/10.1093/nar/ gkr245

Dwyer DJ, Kohanski MA, Hayete B, Collins JJ (2007) Gyrase inhibitors induce an oxidative damage cellular death pathway in Escherichia coli. Mol Syst Biol. https://doi.org/10.1038/msb4100135

Dwyer DJ, Belenky PA, Yang JH et al (2014) Antibiotics induce redoxrelated physiological alterations as part of their lethality. Proc Natl Acad Sci USA 111:E2100-E2109. https://doi.org/10.1073/ pnas. 1401876111

Fang FC (1997) Mechanisms of nitric oxide-related antimicrobial activity. J Clin Invest 99:2818-2825. https://doi.org/10.1172/ JCI119473

Farha MA, French S, Stokes JM, Brown ED (2018) Bicarbonate alters bacterial susceptibility to antibiotics by targeting the proton motive force. ACS Infect Dis 4:382-390. https://doi.org/10.1021/acsinfecdi s.7b00194

Forde BM, Ben Zakour NL, Stanton-Cook M et al (2014) The complete genome sequence of Escherichia coli EC958: a high quality reference sequence for the globally disseminated multidrug resistant E. coli O25b:H4-ST131 clone. PLoS ONE 9:e104400. https://doi. org/10.1371/journal.pone. 0104400

Foti JJ, Devadoss B, Winkler JA et al (2012) Oxidation of the guanine nucleotide pool underlies cell death by bactericidal antibiotics. Science 336:315-319. https://doi.org/10.1126/science.1219192

Hart TW (1985) Some observations concerning the S-nitroso and S-phenylsulfonyl derivatives of L-cysteine and glutathione. Tetrahedron Lett 26:2013-2016. https://doi.org/10.1016/S0040-4039(00)98368 $-0$

Imlay JA, Fridovich I (1991) Superoxide production by respiring membranes of Escherichia coli. Free Radic Res Commun 12-3:59-66. https://doi.org/10.3109/10715769109145768

Imlay JA, Chin SM, Linn S (1988) Toxic DNA damage by hydrogen peroxide through the Fenton reaction in vivo and in vitro. Science 240:640-642. https://doi.org/10.1126/science.2834821

Jia B, Raphenya AR, Alcock B et al (2017) CARD 2017: expansion and model-centric curation of the comprehensive antibiotic resistance database. Nucleic Acids Res 45:D566-D573. https://doi. org/10.1093/nar/gkw1004

Johnson JR, Johnston B, Clabots C et al (2010) Escherichia coli sequence type ST131 as the major cause of serious multidrug-resistant $E$. coli infections in the United States. Clin Infect Dis 51:286-294. https:// doi.org/10.1086/653932

Keszler A, Zhang Y, Hogg N (2010) Reaction between nitric oxide, glutathione, and oxygen in the presence and absence of protein: How are S-nitrosothiols formed? Free Radic Biol Med 48:55-64. https:// doi.org/10.1016/j.freeradbiomed.2009.10.026

Keyer K, Imlay JA (1996) Superoxide accelerates DNA damage by elevating free-iron levels. Proc Natl Acad Sci U S A 93:13635-13640. https://doi.org/10.1073/pnas.93.24.13635

Kohanski MA, Dwyer DJ, Hayete B et al (2007) A common mechanism of cellular death induced by bactericidal antibiotics. Cell 130:797-810

Kohanski MA, Dwyer DJ, Collins JJ (2010) How antibiotics kill bacteria: from targets to networks. Nat Rev Microbiol 8:423-435

Letunic I, Bork P (2016) Interactive tree of life (iTOL) v3: an online tool for the display and annotation of phylogenetic and other trees. Nucleic Acids Res 44:W242-W245. https://doi.org/10.1093/nar/ gkw290

Lobritz MA, Belenky P, Porter CBM et al (2015) Antibiotic efficacy is linked to bacterial cellular respiration. Proc Natl Acad Sci USA 112:8173-8180. https://doi.org/10.1073/pnas. 1509743112
Maiden MCJ, Bygraves JA, Feil E et al (1998) Multilocus sequence typing: a portable approach to the identification of clones within populations of pathogenic microorganisms. Proc Natl Acad Sci USA 95:3140-3145. https://doi.org/10.1073/pnas.95.6.3140

McArthur AG, Waglechner N, Nizam F et al (2013) The comprehensive antibiotic resistance database. Antimicrob Agents Chemother 57:3348-3357. https://doi.org/10.1128/AAC.00419-13

McCollister BD, Hoffman M, Husain M, Vazquez-Torres A (2011) Nitric oxide protects bacteria from aminoglycosides by blocking the energy-dependent phases of drug uptake. Antimicrob Agents Chemother 55:2189-2196. https://doi.org/10.1128/aac.01203-10

Mobley HL, Green DM, Trifillis AL et al (1990) Pyelonephritogenic Escherichia coli and killing of cultured human renal proximal tubular epithelial cells: role of hemolysin in some strains. Infect Immun 58:1281-1289. https://doi.org/10.1128/IAI.58.5.1281-1289.1990

Nicolas-Chanoine M-H, Blanco J, Leflon-Guibout V et al (2008) Intercontinental emergence of Escherichia coli clone O25: H4-ST131 producing CTX-M-15. J Antimicrob Chemother 61:273-281. https ://doi.org/10.1093/jac/dkm464

Nicolas-Chanoine M-H, Bertrand X, Madec J-Y (2014) Escherichia coli ST131, an intriguing clonal group. Clin Microbiol Rev 27:543-574. https://doi.org/10.1128/CMR.00125-13

Notredame C, Higgins DG, Heringa J (2000) T-coffee: a novel method for fast and accurate multiple sequence alignment. J Mol Biol 302:205217. https://doi.org/10.1006/jmbi.2000.4042

Okonechnikov K, Golosova O, Fursov M (2012) Unipro UGENE: a unified bioinformatics toolkit. Bioinformatics 28:1166-1167. https:// doi.org/10.1093/bioinformatics/bts091

Page AJ, Cummins CA, Hunt M et al (2015) Roary: rapid large-scale prokaryote pan genome analysis. Bioinformatics 31:3691-3693. https://doi.org/10.1093/bioinformatics/btv421

Price MN, Dehal PS, Arkin AP (2009) FastTree: computing large minimum evolution trees with profiles instead of a distance matrix. Mol Biol Evol 26:1641-1650. https://doi.org/10.1093/molbev/msp077

Reffuveille F, de la Fuente-Nunez C, Fairfull-Smith KE, Hancock REW (2015) Potentiation of ciprofloxacin action against Gram-negative bacterial biofilms by a nitroxide. Pathog Dis. https://doi.org/10.1093/ femspd/ftv016

Roos V, Ulett GC, Schembri MA, Klemm P (2006) The asymptomatic bacteriuria Escherichia coli strain 83972 outcompetes uropathogenic E. coli strains in human urine. Infect Immun 74:615-624. https:// doi.org/10.1128/iai.74.1.615-624.2006

Sapri HF, Sani NAM, Neoh H-M, Hussin S (2013) Epidemiological study on Staphylococcus aureus isolates reveals inverse relationship between antibiotic resistance and virulence repertoire. Indian J Microbiol 53:321-322. https://doi.org/10.1007/s12088-013-0357-4

Seemann T (2014) Prokka: rapid prokaryotic genome annotation. Bioinformatics 30:2068-2069. https://doi.org/10.1093/bioinformatics/ btu153

Shepherd M, Achard MES, Idris A et al (2016) The Cytochrome $b d$-I terminal oxidase of uropathogenic Escherichia coli augments survival during infection. Sci Rep 6:35285. https://doi.org/10.1038/ srep35285

Stokes JM, Lopatkin AJ, Lobritz MA, Collins JJ (2019) Bacterial metabolism and antibiotic efficacy. Cell Metab 30:251-259. https://doi. org/10.1016/j.cmet.2019.06.009

Taber HW, Mueller JP, Miller PF, Arrow AS (1987) Bacterial uptake of aminoglycoside antibiotics. Microbiol Rev 51:439-457

Totsika M, Kostakioti M, Hannan TJ et al (2013) A FimH inhibitor prevents acute bladder infection and treats chronic cystitis caused by multidrug-resistant uropathogenic Escherichia coli ST131. J Infect Dis 208:921-928. https://doi.org/10.1093/infdis/jit245

Walsh TR, Weeks J, Livermore DM, Toleman MA (2011) Dissemination of NDM-1 positive bacteria in the New Delhi environment and its implications for human health: an environmental point prevalence 
study. Lancet Infect Dis 11:355-362. https://doi.org/10.1016/S1473 -3099(11)70059-7

Weigel LM, Steward CD, Tenover FC (1998) gyrA mutations associated with fluoroquinolone resistance in eight species of Enterobacteriaceae. Antimicrob Agents Chemother 42:2661-2667. https://doi. org/10.1128/aac.42.10.2661

Welch RA, Burland V, Plunkett G et al (2002) Extensive mosaic structure revealed by the complete genome sequence of uropathogenic Escherichia coli. Proc Natl Acad Sci USA 99:17020-17024

Yong D, Toleman MA, Giske CG et al (2009) Characterization of a new metallo-beta-lactamase gene, bla(NDM-1), and a novel erythromycin esterase gene carried on a unique genetic structure in Klebsiella pneumoniae sequence type 14 from India. Antimicrob Agents Chemother 53:5046-5054. https://doi.org/10.1128/AAC.00774-09

Zankari E, Hasman H, Cosentino S et al (2012) Identification of acquired antimicrobial resistance genes. J Antimicrob Chemother $67: 2640-2644$

Publisher's Note Springer Nature remains neutral with regard to jurisdictional claims in published maps and institutional affiliations. 